\title{
Systemic fungal infections in neonates
}

\author{
P C Ng
}

Fungal infection in newborn babies can present as mild, superficial mucocutaneous infection or as life threatening and disseminated sepsis. There are a number of ways in which fungal infection may be acquired. The infection may present at birth as congenital illness or later in life as nosocomial acquired infection.

\section{(I) Acquired systemic fungal infections} Despite improvement in survival rates of very low birthweight (VLBW) infants, nosocomial infection remains an important contributing factor to neonatal morbidity and mortality. The emergence of fungi as important pathogens is likely to be the result of changes in the neonatal intensive care environment and their interaction with the host.

\section{INCIDENCE}

The incidence of disseminated fungal infection has not been systematically reported, due to problems in early recognition of the non-specific clinical symptoms and signs, ${ }^{1}$ and difficulty in confirming the diagnosis by laboratory tests. ${ }^{2}$ Current serological tests are not entirely reliable ${ }^{2}$ and microbiological cultures from sterile body fluids may only be intermittently positive, and even positive results may be misinterpreted as colonisation or contamination. ${ }^{1}$ A significant proportion of cases are only diagnosed at necropsy and those cases without postmortem examination would have been missed. Based on these limitations the incidence of acquired systemic fungal infections is estimated to be $2 \%$ to $5 \%$ among the VLBW infants, ${ }^{3-5}$ with species of candida being the most frequent invaders. ${ }^{46}$

\section{FACTORS PREDISPOSING TO SYSTEMIC FUNGAL} INFECTION

Factors responsible for emergence of fungal infections in modern neonatal intensive care unit include impaired host defence, aggressive neonatal intensive care procedures, and prolonged use of broad spectrum antimicrobial treatment.

\section{Department of Paediatrics, Chinese University of Hong Kong}

(a) Impaired host defence

The preterm infants' fragile skin and mucous membranes are very susceptible to contact injury which frequently results in the invasion of micro-organisms into deeper tissues. Specific immunological defence systems are also underdeveloped and transplacentally acquired maternal IgG is low. ${ }^{7} \mathrm{~T}$ lymphocytes are reduced in number and are functionally incompetent with impaired cytotoxic activity. Both phagocytic and bactericidal activities of polymorphonuclear leucocytes are subnormal. ${ }^{8}$ The monocyte-macrophage system is immature with greatly suppressed chemotactic activity $^{8}$ and inadequate nutrition undoubtedly further compromises immune status.

Immunocompetent term infants very rarely develop systemic fungal infections and they appear to have different risk factors from premature neonates. Most cases are associated with major congenital anomalies that include omphalocele, malrotation, sacrococcygeal teratoma, giant haemangioma, and congenital cyanotic heart disease. Infants who undergo complex surgery are also at a greater risk.

\section{(b) Intensive care procedures}

Invasive therapeutic and monitoring equipment has become an integral part of modern neonatal intensive care. Transcutaneous monitoring devices and adhesive tapes frequently cause superficial skin damage which facilitates the invasion of micro-organisms. ${ }^{9}$ Endotracheal tubes, urinary catheters, and indwelling vascular lines all bypass the skin barrier and enhance the suspectibility to systemic fungal infection by furnishing a portal of entry directly into the bloodstream. ${ }^{10}$ Although intravascular blood pressure monitoring devices and arterial cannulas may cause disseminated sepsis, the central venous line used for total parenteral nutrition is by far the most important apparatus responsible for fungal septicaemia. ${ }^{146911}$ The administration of lipid emulsion may further increase the risk of fungaemia and narcotising pulmonary vasculitis due to lipophilic fungus. ${ }^{12-14}$ In addition, it has also been suggested that prolonged hospitalisation ${ }^{15}$; prolonged stay in an incubator ${ }^{15}$; extended period of endotracheal intubation ${ }^{6}$; the use of tracheostomies ${ }^{6}$; the requirement of an intraventricular shunt ${ }^{4}$; and the use of lamb's wool, sticking plaster, and transparent semipermeable dressing ${ }^{9}{ }^{15}$ may increase the risk of fungal colonisation and subsequent systemic infection. Equipment such as blood pressure cuffs, thermometers, oxygen saturation probes, and other diagnostic devices that are shared between infants may also promote the incidence of cross infection. ${ }^{13}$ 


\section{(c) Drugs}

The suppression of the normal bacterial flora by prolonged and extensive use of broad spectrum antibiotics in VLBW infants has undoubtedly contributed to the increased incidence of fungal sepsis in sick premature infants. ${ }^{14613}$ Theophylline has been reported to inhibit the candidicidal activity of human granulocytes. ${ }^{16}$ The liberal use of dexamethasone in bronchopulmonary dysplasia, however, is not associated with a significant increase in the incidence, or a change in the pattern, of infection. ${ }^{10}$ It seems that the antiinflammatory potency of a particular steroid has little influence on its immunosuppressive action.

\section{PATHOGENS}

Candida and malassezia species are the two most frequent groups of opportunistic organisms causing disseminated fungal infection in premature neonates under 1500 g. $^{46}$ Other opportunistic fungi such as aspergillus, cryptococcus, mucor, rhizopus, saccharomyces, Trichosporm beigelii, etc, are uncommon and usually occur sporadically. Pathogenic fungi which include coccidioides, paracoccidioides, and blastomyces very rarely give rise to neonatal infections outside their endemic areas and are not a major problem in most neonatal units.

\section{CANDIDA SPP}

Candida albicans is the most frequent cause of fungaemia in premature VLBW infants. Other species of candida such as Candida parapsilosis, Candida tropicalis, Candida glabrate, and Candida lusitaniae have been reported to give a clinical picture indistinguishable from that caused by $C$ albicans.

In most preterm infants, colonisation of candida occurs in utero or during delivery ${ }^{17}$ either by swallowing and aspirating the contaminated liquor, or by physical contact with the birth canal. Skin colonisation is common and often precedes the dissemination process. ${ }^{18}$ Infants with oropharyngeal and gastrointestinal tract colonisation may rarely have invasion of the bowel mucosa with resultant fungaemia. ${ }^{19}$ Candida pulmonitis secondary to aspiration is also uncommon. ${ }^{11}$ In contrast, babies colonised with candida after 2 weeks of age usually acquired the fungus nosocomially from caretakers. ${ }^{17}$

\section{(a) Clinical presentation}

The clinical features of systemic candidiasis in neonates are often non-specific and indistinguishable from bacterial sepsis. The onset is usually insidious and the mean age of infection is 33 days. ${ }^{1}$ The infant presents with temperature instability, carbohydrate intolerance, hypotension, apnoea, bradycardia, and deteriorating respiratory function which frequently requires ventilatory support. Feed intolerance, guaiac positive stools and generalised abdominal distension (without pneumatosis intesti- nalis) may also occur. This spectrum of symptoms and signs may be intermittent and may even be absent in some infants. ${ }^{1}$ It seems that a septic VLBW infant who deteriorates despite antibiotic treatment should always be considered to have systemic fungal infection especially when recognisable predisposing factors are present. In contrast, those cases diagnosed at necropsy tend to have had their initial presentation at a much earlier age, with fewer predisposing factors, and have followed a fulminant downhill course.

\section{(b) Deep organ involvement}

Deep organ involvement is commonly associated with systemic candidiasis. Candida spp have a high affinity for the renal tract. Haematuria, proteinuria, and pyuria are usually present with candiduria. Acute oliguria is associated with candida pyelonephritis, renal papillary necrosis, multiple parenchymal abscesses, and obstructing fungal balls. ${ }^{20-22}$ Secondary hypertension due to renal artery obstruction is rare. ${ }^{21}$ Candida can infiltrate the eyes via the haematogenous route causing endophthalmitis. ${ }^{15}$ These lesions are white, cotton wool ball-like and rapidly progress to involve the vitreous humour. Early detection of these lesions is important because they can cause permanent blindness. Septic arthritis ${ }^{23} 24$ and osteomyelitis ${ }^{25}$ are uncommon manifestations. Such infants usually present with a red, warm, tender, swollen, and fluid filled joint with the affected limb typically placed in an abnormal posture. The knee is the most frequent joint to be involved. It has been suggested that catheterisation of the umbilicus colonised with candida gives rise to fungaemia and subsequent synovial infection. ${ }^{23}$ Intracardiac fungal masses and endocarditis are frequently associated with indwelling right atrial lines. ${ }^{626}$ The fungal masses may obstruct the venous return to the heart ${ }^{24} 26$ giving rise to persistent peripheral oedema, hepatosplenomegaly, and the superior vena cava syndrome. Multiple septic emboli can occur leading to a wider dissemination of the infection. Pulmonary candidiasis is difficult to diagnose during life. Nodular infiltrates, focal cavitation, and progressive air space consolidation are the most frequent but non-specific radiological abnormalities observed. ${ }^{27}$ Candida may also gain entry into the bloodstream by invading the bowel mucosa. ${ }^{19}$ Fungal necrotising enterocolitis is usually severe and carries a poor prognosis. Central nervous system involvement is most commonly associated with intraventricular shunts. ${ }^{424}$ Meningitis, ventriculitis, and multiple cerebral abscesses have been described. ${ }^{24} 28$

\section{(c) Diagnosis and investigations}

The isolation of Candida spp from blood culture is the most important investigation for establishing the diagnosis of disseminated candidiasis. Blood samples may be taken from the indwelling central line, peripheral vein or artery. Although a positive blood culture is 
nearly always significant, a negative culture does not exclude the diagnosis, as up to $20 \%-50 \%$ may not grow the organism. ${ }^{6}$ It must be emphasised that fungus grows more slowly than bacteria and antifungal treatment must therefore not be withheld in the absence of positive culture when clinical evidence is overwhelming. Examination of a Gram stained peripheral blood buffy coat preparation is sometimes useful for rapid diagnosis. ${ }^{29}$ The presence of budding yeast and hyphae in a suprapubic urine sample is diagnostic of deep seated fungal infection. Culture from other sterile sites such as cerebrospinal fluid, ascites fluid, pleura, and tissue biopsy together with culture of indwelling catheters and long lines should be obtained when clinically indicated. Candida meningitis is difficult to diagnose. Despite an increase in the cerebrospinal fluid white cell count and protein concentration, microscopy is usually negative. Moreover, these parameters may also be increased with periventricular haemorrhage without fungal infection in the central nervous system. Surveillance cultures from ears, throat, umbilicus, and rectum may also be helpful in identifying the colonisation pattern of the individual patient.

Detection of candida antigens using various enzyme linked immunosorbent assay techniques appeared to be promising with a diagnostic sensitivity of about $70 \%$ when serial specimens are evaluated. ${ }^{23031}$ However, serum levels of candida antigen can be raised in individuals who are heavily colonised or be undetectable in those with proved systemic candidiasis. The polymerase chain reaction technique, with its capability of detecting candida DNA fragments, may in future represent an important breakthrough for the early detection of candida sepsis. Measurement of candida metabolites such as D-arabinitol and biochemical tests such as the API-20c yeast test are of limited value and not routinely used in laboratories. ${ }^{2}$

White cell and platelet counts are often abnormal in candida septicaemia. Serial measurements of $\mathrm{C}$ reactive protein are particularly useful in monitoring the response to treatment. ${ }^{929}$ A chest radiograph may show pulmonary infiltrates and progressive air space consolidation but these changes are rather non-specific and not unique for candida pulmonitis.

Specific investigations are sometimes used to locate the focus of infection. Retinal examination may reveal fluffy white lesions on retina or vitreous, interlesional and lesional strands, and diffuse vitreous haze. ${ }^{1}$ Evidence of ventriculitis with foci of cavitary destruction and a network of fungal strands in the ventricles may be apparent on cranial ultrasound scan. ${ }^{32}$ Cardiac and renal ultrasound scans should be routinely performed. Intracardiac fungal infection may present as thrombi in the heart chambers and vegetations on the valves, ${ }^{24} 26$ whereas renal involvement may manifest as enlarged kidneys with highly echogenic parenchyma and fungal balls within the dilated pelvicalyceal systems. ${ }^{32}$ Percutaneous needle aspiration of the synovial cavity is indicated when neonatal fungal arthritis is suspected. ${ }^{23}$

\section{(d) Treatment}

Three main categories of antifungal drugs are currently used for the treatment of systemic fungal infections. They are the polyene macrolide class compounds, the azoles, and inhibitors of RNA synthesis, the fluorinated pyrimidines.

Amphotericin B belongs to the polyene macrolide class of drugs and is the mainstay of treatment for opportunistic fungal infection. It must be administered intravenously for systemic effect as it is not absorbed enterally. The dosage could be increased in a stepwise fashion starting from $0.25 \mathrm{mg} / \mathrm{kg} /$ day up to a maximum of $1 \mathrm{mg} / \mathrm{kg} /$ day or administered as a constant dose of $0.6 \mathrm{mg} / \mathrm{kg} /$ day throughout the treatment period. The usual recommended duration of treatment is between three to six weeks. Combining amphotericin B treatment with flucytosine will permit the use of a lower dose and this combination is particularly effective for the treatment of central nervous system candida infection. ${ }^{46}$ Although the side effects are better tolerated by infants, fever, renal toxicity, gastrointestinal upset, bone marrow suppression, anaphylactic reaction, and severe electrolytes disturbances including hypokalaemia and hypomagnesaemia have been well documented. Electrolytes and renal and hepatic function must be monitored closely during the treatment period. Preparation of amphotericin B with parenteral lipid solution has been reported to reduce nephrotoxicity, improves clinical tolerance and permits a larger daily dose to be used. ${ }^{33}$

A new formulation of amphotericin encapsulated in liposomes is commercially available. The resultant liposomal preparation is designed to maximise the delivery of amphotericin to deep seated sites of infection. The uptake by macrophages and the transport of liposomal amphotericin to the infective areas appear to play a major part in increasing efficacy and diminishing toxicity. ${ }^{34} 35$ Two reports indicate that liposomal amphotericin is effective in the treatment of disseminated fungal infections in VLBW infants. ${ }^{3637} \mathrm{~A}$ starting dose of $1.5 \mathrm{mg} / \mathrm{kg} /$ day was used and this was increased progressively over a week to a maximum of $5 \mathrm{mg} / \mathrm{kg} /$ day. ${ }^{36}$ Side effects associated with conventional amphotericin such as fever, bronchospastic reactions, and renal toxicity were significantly less, ${ }^{36} 37$ but neonatal cholestasis with raised hepatic enzymes values and conjugated hyperbilirubinaemia has been observed. ${ }^{37}$ High dose liposomal amphotericin should be tried when the fungal infection is refractory or the baby is unable to tolerate conventional treatment.

The azole group of antifungals include the imidazoles such as miconazole, ketoconazole, and the newer triazoles such as fluconazole, itraconazole, and saperconazole. They are fungalstatic agents. All triazoles show promise as broad spectrum, orally active, systemic antifungal drugs with less toxicity than the 
imidazoles. Fluconazole has been successfully used with minimal adverse effects ${ }^{4}$ for the treatment of neonatal candida septicaemia, ${ }^{38}$ pneumonia, ${ }^{39}$ osteomyelitis, ${ }^{25}$ central nervous system shunt infection, 40 and pelviureteric candidal bezoars. ${ }^{22} \mathrm{~A}$ dose of $6 \mathrm{mg} / \mathrm{kg} /$ day is commonly used. A recent study suggested that an adequate drug concentration in the blood could be achieved using a regimen of $6 \mathrm{mg} / \mathrm{kg}$ every three days in the first week followed by $6 \mathrm{mg} / \mathrm{kg}$ every two days or even daily in subsequent weeks, as the half life of fluconazole varies from 55 to 89 hours in VLBW babies. ${ }^{41}$ Despite good evidences of its therapeutic efficacy, 22 25 38-40 treatment failure in the presence of adequate minimum inhibitory concentrations has been reported. ${ }^{42}$ This group of drugs should, therefore, be considered as second line treatment for infants who do not respond to the standard treatment or whose condition does not permit the use of toxic antifungal agents.

Flucytosine is a synthetic compound and has the advantages of excretion via the urinary tract and good absorption when given orally. Synergy has been demonstrated with amphotericin B. ${ }^{43}$ Flucytosine is not recommended to be used as a single agent because resistance is a common problem and can develop during treatment. Flucytosine is given in a dose of 100 $\mathrm{mg} / \mathrm{kg} / \mathrm{day}^{44}$ and serum drug concentrations and blood counts must be monitored at least once a week because it can induce severe bone marrow suppression. Other side effects are mild and include gastrointestinal, hepatic, and renal impairment.

Acute resuscitation with plasma expander, inotropic agents, and mechanical ventilatory support are often required in the acute phase of systemic fungal illness. Removal of the infected indwelling lines ${ }^{9}$ and intraventricular shunt are mandatory. Surgical drainage of subcutaneous fungal abscess can be carried out at the bedside under local anaesthesia. ${ }^{9}$ Consultation with the orthopaedic team will help to define the situation when surgical drainage of the septic joint and convalescent immobilisation in a plaster cast is necessary. Urinary diversion, local irrigation with amphotericin $B$, and guidewire fragmentation of renal fungal balls have been attempted. ${ }^{45}$ The indications for surgical removal of intracardiac fungal masses will depend on the response to antifungal treatment, the size and mobility of the mass, the likelihood of embolisation and its haemodynamic significance on the patient. For infants under $2000 \mathrm{~g}$ in weight, cardiopulmonary bypass is technically difficult and risky. The removal of intracardiac masses has been successful with the use of an inflow occlusion technique. ${ }^{26}$

\section{(e) Prognosis}

Disseminated candida infections are associated with high morbidity and mortality in neonates with a significant proportion of cases being diagnosed at necropsy. Although central nervous system involvement usually has a poor prognosis and frequent neurological sequelae, intact survivors are occasionally seen. ${ }^{46}$ Current mortality rates range from $18 \%{ }^{46}$ to $50 \%,{ }^{128}$ but with a trend towards prompt and aggressive antifungal treatment, outcome should improve.

\section{(f) Prevention}

Since the colonisation of the newborn with subsequent mucocutaneous candidiasis may predispose to systemic candida infection, attempts have been made to eradicate the organism from the skin and gastrointestinal tract of at risk infants. Clotrimazole vaginal tablets and cream given to pregnant women with vaginal candidiasis have significantly reduced the number of fungal contaminated newborns. ${ }^{47}$ Breast feeding has no impact on the incidence of oral thrush nor has chlorhexidine alcohol spray applied to the nipples of breast feeding women. The most promising prophylactic measure is perhaps the use of oral antifungal drugs, namely nystatin and miconazole oral gel, which have been shown to be effective in reducing the incidence of postnatally acquired gastrointestinal fungal colonisation. ${ }^{48} 49$ Nevertheless, the success of oral antifungal prophylaxis has not been translated into prevention of systemic candida infection in the newborns. As disseminated fungal infection is becoming more frequent among preterm neonates, there must be stringent control in the selection and usage of antibiotics, judicious use of indwelling lines and expedient weaning from mechanical ventilation. These must be combined with strict implementation of infection control surveillance measures in the intensive care nursery and especially careful handwashing. ${ }^{50}$

\section{MALASSEZIA SPP}

Malassezia furfur is a lipophilic yeast. It is a frequent skin commensal and may colonise up to $64 \%$ to $84 \%$ of babies in a neonatal unit. ${ }^{1551}$ Bronchopneumonia with necrotising pulmonary vasculitis and fungaemia in infants receiving parenteral lipid therapy have been reported. ${ }^{12-14} M$ furfur cannot be recovered from routine culture medium because it has an absolute nutritional requirement for medium chain fatty acids $\left(\mathrm{C}_{12}-\mathrm{C}_{24}\right) .{ }^{4613}$ Lipid enriched medium (such as Sabourand's dextrose agar with olive oil and $0 \cdot 2 \%$ Tween 80 ) is required for its culture. ${ }^{4}$ As most cases resolve quickly after parenteral lipid is stopped and indwelling venous catheter is removed, only a short course of amphotericin B is required. The prognosis is usually good if the diagnosis is made early and appropriate measures promptly implemented.

\section{(2) Congenital fungal infections}

In the United States $5 \%$ to $10 \%$ of fetal deaths each year are due to intrauterine infections. ${ }^{2}$ Diagnosis of fetal infection is often difficult and requires elaborate laboratory tests for isolation of the organism or demonstration of its specific nucleic acids. Congenital fungal 
infection is extremely rare but has been reported with candida, blastomyces, coccidioides, and cryptococcus.

The syndrome of congenital systemic candida infection is very different from the more common acquired infection via haematogenous dissemination. Predisposing factors such as previous exposure to broad spectrum antibiotics, total parenteral nutrition, and central venous catheterisation are typically absent. The presence of a foreign body in the mother's genital tract such as an intrauterine contraceptive device or a cervical suture contributes to colonisation of Candida spp. ${ }^{52-54}$ Although severe candidal sepsis with pneumonia, ${ }^{52}$ septicaemia, and meningitis ${ }^{3}$ have been reported, the pattern of organ involvement usually suggests a slow, locally invasive infection with little evidence of haematogenous dissemination, even in cases with deep tissue infection. ${ }^{53}$ Congenital candidiasis typically presents in the first week of life and may simulate congenital infection of bacterial origin or other conditions such as respiratory distress syndrome or idiopathic persistent pulmonary hypertension of the newborn. In most cases, candida can be recovered from surface swabs, endotracheal or gastric aspirates. Histological examination and culture of the placenta and umbilical cord may provide important evidences for the diagnosis. ${ }^{2}$ Blood and cerebrospinal fluid specimens, however, frequently fail to demonstrate the infection despite the presence of serious generalised candidiasis. ${ }^{53}$ Antifungal treatments are similar to postnatally acquired systemic candidiasis. Congenitally infected infants have a high mortality. ${ }^{53}$

\section{Summary}

Systemic fungal infections, previously considered to be a rare complication, are now frequently diagnosed in VLBW infants receiving intensive care. Confirming the diagnosis by laboratory tests is difficult and a high index of suspicion is required. Prompt and aggressive use of antifungal treatment is justified in a clinically septic neonate, especially those with a raised serum concentration of $\mathrm{C}$ reactive protein, who do not show a satisfactory response to antibiotics. The newer generation of liposomal amphotericin and azole antifungal drugs appear to be safe, effective, and well tolerated. With increasing awareness, prompt treatment, and better neonatal intensive care, the outcome of systemic fungal infection in preterm infants should improve.

1 Baley JE, Kliegman RM, Fanaroff AA. Disseminated fungal infections in very low birth weight infants: clinical manifestations and epidemiology. Pediatrics 1984; 73: 144-52.

2 McGowan KL, Hodinka RL. Laboratory diagnosis of fetal infections. Clin Lab Med 1992; 12: 523-52.

3 Butler KM, Baker CJ. Candida: an increasingly important pathogen in the nursery. Pediatr Clin North Am 1988; 35: 543-63.

4 Phillips G, Golledge C. Fungal infection in neonates. f Antimicrob Chemother 1991; 28: 159-61.

5 Baley JE, Annable WL, Kliegman RM. Candida endophthalmitis in the premature infant. $\mathcal{F}$ Pediatr 1981; 98: 458-61.

6 Nicholls JM, Yuen KY, Tam AY. Systemic fungal infections in neonates. Br f Hosp Med 1993; 49: 420-4.
7 Conway SP, Ng PC, Howel D, Maclain B, Gooi HC. Prophylactic intravenous immunoglobulin in preterm Prophylactic intravenous immunoglobulin in prete

8 Pearse RG, Roberton NRC. Infection in the newborn. In: Roberton NRC, ed. Textbook of neonatology. 1 1st Ed. Edinburgh: Churchill Livingstone, 1986: 725-81.

$9 \mathrm{Ng}$ PC, Dear PRF. Phycomycotic abscesses in a preterm infant. Arch Dis Child 1989; 64: 862-4.

$10 \mathrm{Ng}$ PC, Thomson MA, Dear PRF. Dexamethasone and infection in preterm babies: a controlled study. Arch Dis Child 1990; 65: 54-8.

11 Baley JE. Neonatal candidiasis: the current challenge. Clin Perinatol 1991; 18: 263-80.

12 Redline RW, Dahm BB. Malassezia pulmonary vasculitis in an infant on long-term Intralipid therapy. $N$ Engl $\mathcal{f}$ Med 1981; 305: 1395-8.

13 Richet HM, McNeil MM, Edwards MC, Javis WR. Cluster of Malassezia furfur pulmonary infection in infants in a neonatal intensive-care unit. ff Clin Microbiol 1989; 27: $1197-200$

14 Dankner WM, Spector SA, Fierer J, Davis CE. Malassezia fungemia in neonates and adults: complication of hyperalimentation. Rev Infect Dis 1987; 9: 743-53.

15 Powell DA, Hayes J, Durrell DE, Miller M, Marcon MJ. Malassezia fufur skin colonization of infants hospitalized in intensive care units. $\mathcal{F}$ Pediatr $1987 ; 111: 217-20$.

16 Bourne HR, Lehrer RI, Cline MJ. 3' $5^{\prime}$-cyclic adenosine monophosphate in the human leukocyte: synthesis, degradation, and effects on neutrophil candidacidal activity. fClin Invest 1971; 50: 920-9.

17 Baley JE, Kliegman RM, Boxerbaum B, Fanaroff AA. Fungal colonization in the very low birth weight infants. Pediatrics 1986; 78: 225-32.

18 Faix RG, Kovarik SM, Shaw TR, Johnson RV. Mucocutaneous and invasive candidiasis among very low birth weight ( $\mu 1500$ grams) infant in intensive care nurseries: a prospective study. Pediatrics 1989; 83: 101-7

19 Keller MA, Sellers BB, Melish ME, Kaplan GW, Miller $\mathrm{KE}$, Mendoza SA. Systemic candidiasis in infants: a case presentation and literature review. Am F Dis Child 1977; 131: $1260-3$.

20 Tomashefski JF, Abramowsky CR. Candida-associated renal papillary necrosis. Am f Clin Pathol 1981; 75:190-4.

21 Heckmatt JZ, Meadow SR, Anderson CK. Acute anuric renal failure in an infant with systemic candidiasis. Arch Dis Child 1979; 54: 70-2.

22 Bergman KA, Meis JF, Horrevorts AM, Monnens L. Acute renal failure in a neonate due to pelviureteric candidal bezoars successfully treated with long-term systemic fluconazole. Acta Paediatr 1992; 81: 709-11.

23 Pittard WB, Thullen JD, Fanaroff AA. Neonatal septic arthritis. F Pediatr 1976; 88: 621-4.

24 Ho NK. Systemic candidiasis in premature infants. Australian Paediatric fournal 1984; 20: 127-30.

25 Oleinik EM, Della-Latta P, Rinaldi MG, Saiman L. Candida lusitaniae osteomyelitis in a premature infant. Am f Perinatol 1993; 10: 313-5.

26 Foker JE, Bass JL, Thompson T, Tilleli JA, Johnson DE. Management of intracardiac fungal mass in premature Management of intracardiac fungal mass in prem
infants. $\mathcal{F}$ Thorac Cardiovasc Surg 1984; 87: 244-50.

27 Kassner KG, Kauffman SL, Yoon JJ, Semiglia M, Kozinn PJ, Goldberg PL. Pulmonary candidiasis in infants: clinical, radiologic, and pathologic features. Am $\mathcal{f}$ Radiol 1981; 137: 707-16.

28 Faix RG. Systemic candida infections in intensive care nurseries: high incidence of central nervous system involvement. F Pediatr 1984; 105: 616-22.

29 Cattermole HEJ, Rivers RPA. Neonatal candida septicaemia: diagnosis on buffy smear. Arch Dis Child 1987; 62: $302-4$.

30 Barnes RA. Immunological diagnosis of fungal infection in the immunocompromised host. Reviews in Medical Microbiology 1990; 1: 58-65.

31 Jones JM. Laboratory diagnosis of invasive candidiasis. Clin Microbiol Rev 1990; 3: 32-45.

32 Currie JL. Ultrasound appearances of systemic candidiasis in the neonate. Radiography Today 1989; 55: 20-1.

33 Moreau P, Milpied N, Fayette N, Ramee JF, Harousseau JL. Reduced renal toxicity and improved clinical tolerance of amphotericin B mixed with Intralipid compared with conventional amphotericin B in neutropenic patients. f Antimicrob Chemother 1992; 30: 535-41.

34 Lopez-Berestein G, Kasi L, Rosenblum M. Clinical pharmacology of $99 \mathrm{mTc}$-labeled liposomes in patients with cancer. Cancer Res 1984; 44: 375-8.

35 Poffitt R, Satorius A, Chiang S, Sullivan L, Adler-Moore J. Pharmacology and toxicology of a liposomal formulation of amphotericin B (AmBisome) in rodents. F Antimicrob Chemother 1991; 28: 49-61.

36 Lackner $\mathrm{H}$, Schwinger W, Urban $\mathrm{C}$, et al. Liposomal amphotericin B (AmBisome) for treatment of disseminated fungal infections in two infants of very low birth nated fungal infections in two infants

37 da Silva LP, Amaral JM, Ferreiva NC. Which is the most appropriate dosage of liposomal amphotericin-B (AmBisome) for the treatment of fungal infections in infants of very low birth weight? Pediatrics 1993; 91: 1217-8.

38 Bode S, Pedersen-Bjergaard L, Hjelt K. Candida albicans septicaemia in a premature infant successfully treated with oral fluconazole. Scand f Infect Dis 1992; 24: 673-5.

39 Harms K, Herting E, Schiffmann JH, Speer CP. Candida infections in premature infants weighing less than $1500 \mathrm{~g}$. Mucocutaneous colonization and incidence of systemic infections. Monatsschr Kinderheilkd 1992; 140: 633-8. 
40 Cruciani M, Di Perri G, Molesini M, Vento S, Concia E, Bassetti D. Use of fluconazole in the treatment of Candida albicans hydrocephalus shunt infection. Eur $f$ Clin Microbiol Infect Dis 1992; 11: 957.

41 Saxen H, Hoppu K, Pohjavuori M. Pharmacokinetics of fluconazole in very low birth weight infants durin the first 2 weeks of life. Clin Pharmacol Ther 1993; 54: 269-77.

42 Colville $\dot{A}$, Wale MCJ. Fluconazole or amphotericin for candidaemia in non-neutropenic patients? Lancet 1991; 337: $1605-6$.

43 Polak A. Combination therapy with antifungal drugs. Mykoses 1988; 31 (suppl 2): 45-53.

44 Baley JE, Meyers C, Kleigman RM, Jacobs MR, Blumer JL. Pharmacokinetic outcome of treatment and toxic effects of amphotericin $\mathrm{B}$ and 5 fluorocytosine in neonates. of Pediatr 1990; 116: 791-7.

45 Bell DA, Rose SC, Starr NK, Jaffe RB, Miller FJ Jr. Percutaneous nephrostomy for non-operative management of fungal urinary tract infections. $\mathcal{f}$ Vasc Interv Radio 1993; 4: 311-5.

46 Loke HL, Verber I, Szymonowicz W, Yu VYH. Systemic candidiasis and pneumonia in preterm infants. Australian Paediatric fournal 1988; 24: 138-42.
47 Ruiz Velasco V, Rosas-Arceo J. Prophylactic clotrimazole treatment to prevent mycoses contamination of the newborn. Int $f$ Gynaecol Obstet 1978; 16: 70-1.

48 Sims ME, Yoo Y, You H, Salminen C, Walther FJ. Prophylactic oral nystatin and fungal infections in very-low-birthweight infants. Am $\mathcal{f}$ Perinatol 1988; 5: very- $33-6$

49 Wainer S, Cooper PA, Funk E, Bental RY, Sandler DA Patel J. Prophylactic miconazole oral gel for the prevention of neonatal fungal rectal colonization and systemic infection. Pediatr Infect Dis f 1992; 11: 713-6.

50 Burnie JP, Lee W, Williams JD, Matthews RC, Odds FC. Control of an outbreak of systemic Candida albicans. BMF 1985; 291: 1012-3.

51 Marcon MJ, Powell DA. M furfur. Clinical Microbiology Newsletter 1988; 10: 41-8.

52 Mamlok RI, Richardson CJ, Mamlok V, Nichols MM Goldblum RM. A case of intrauterine pulmonary candidiGoldblum RM. A case of intrauterine pu

53 Whyte RK, Hussain Z, deSa D. Antenatal infections with candida species. Arch Dis Child 1982; 57: 528-35.

54 Baley JE, Silverman R. Systemic candidiasis: cutaneous manifestations in very low birth weight infants. Pediatrics 1988; 82: 211-5. 